\title{
Reflets
}

Revue ontaroise d'intervention sociale et communautaire

\section{Les pertes, les deuils et le processus de croissance des femmes}

\section{Geneviève M. Côté}

Volume 6, numéro 2, automne 2000

Problèmes sociaux en Ontario français

URI : https://id.erudit.org/iderudit/026318ar

DOI : https://doi.org/10.7202/026318ar

Aller au sommaire du numéro

Éditeur(s)

Reflets : Revue ontaroise d'intervention sociale et communautaire

ISSN

1203-4576 (imprimé)

1712-8498 (numérique)

Découvrir la revue

Citer cet article

Côté, G. M. (2000). Les pertes, les deuils et le processus de croissance des femmes. Reflets, 6(2), 117-137. https://doi.org/10.7202/026318ar

Tous droits réservés (C) Reflets : Revue ontaroise d'intervention sociale et communautaire, 2000
Ce document est protégé par la loi sur le droit d'auteur. L'utilisation des services d'Érudit (y compris la reproduction) est assujettie à sa politique d'utilisation que vous pouvez consulter en ligne.

https://apropos.erudit.org/fr/usagers/politique-dutilisation/ 


\section{Les pertes, les deuils et le processus de croissance des femmes $^{1}$}

\section{Geneviève M. Côté}

Travailleuse sociale, Londres, Angleterre

Une croyance populaire veut que le temps guérisse toutes les blessures. Occasionnellement, cela se révèle juste. La personne qui a vécu une perte importante peut se réveiller un jour pour découvrir que ça va mieux. La tempête passe et elle est toujours là, survivante, vivante... voire heureuse. Cependant, tel n'est pas toujours le cas. Malgré le temps qui passe, les chocs peuvent s'accumuler et, pour certaines femmes, il n'y a guère de répit et peu de possibilités de s'en sortir. Comment les femmes surviventelles, compte tenu de la complexité de telles situations? Quels sont les mouvements qui permettent le passage de la souffrance à la guérison? Adoptent-elles des stratégies de survie, de croissance ou de guérison?

Dans cet article, nous explorons la notion de perte qui peut survenir lors d'une déstabilisation tel un décès, une séparation ou une transformation et qui implique une rupture avec quelqu'un ou quelque chose qui nous est cher. Le travail de deuil associé à

"L'objet de cette recherche est de tisser des liens entre le vécu de pertes, le travail de deuil, les stratégies et le processus de guérison des femmes..." ces déstabilisations ou à ces transformations est-il comparable à celui du cheminement que la personne vit lorsqu'elle transige avec ces pertes? L'objet de cette recherche est de tisser des liens entre le vécu de pertes, le travail de deuil, les stratégies et le processus de guérison des femmes selon deux approches peu explorées dans la littérature conventionnelle, soit les perspectives féministe et spirituelle. 
"Au-delà d'un deuil dit "normal "... il y a le cheminement vers la formulation de la perte et éventuellement vers le processus de guérison où la femme vit un changement, une transformation habituellement enrichissante. "
Le domaine médical et scientifique a attribué plusieurs appellations, définitions et symptômes au travail de deuil non résolu, tels que deuils « compliqués », « chroniques » ou «pathologiques ». À ce propos, il sera important de considérer les causes ou les raisons sousjacentes à ces non-résolutions. Au-delà d'un deuil dit «normal »selon nos normes socioculturelles, historiques et médicales-il y a le cheminement vers la formulation de la perte et éventuellement vers le processus de guérison où la femme vit un changement, une transformation habituellement enrichissante.

Le processus de guérison serait-il la dernière phase d'un travail de deuil résolu? Peut-on définir ce dernier concept? Est-il vraisemblable de non seulement accepter la perte, mais aussi d'y trouver un potentiel de croissance? Si oui, quelles sont les pistes possibles? Quels outils ou méthodes les intervenantes peuvent-elles développer pour accompagner les femmes dans de tels cheminements?

Dans un premier temps, nous présentons la thématique de recherche afin de décrire les bases de l'article. Cette partie contient les définitions de certains concepts clés, ainsi qu'une recension d'écrits pertinents. Un résumé de certaines approches théoriques propres à la psychologie et à l'anthropologie offre une amorce de perspective au sujet de l'état des connaissances sur le travail de deuil. L'analyse féministe porte un regard sur les conséquences de certaines pertes et souffrances des femmes dans notre société, ainsi que sur les stratégies de survie et le travail de deuil. L'analyse spirituelle recense des écrits s'intéressant au rôle de la spiritualité dans le deuil et le processus de guérison des femmes. On y explore le concept du vécu de la perte, du deuil et de la guérison dans un passage de la douleur vers la réconciliation. La conclusion propose un résumé du résultat de ces explorations, sous forme de questions et de pistes de réflexion face à l'intervention en vue de recherches futures sur le deuil et la guérison.

\section{Méthodologie}

Cet article présente de façon abrégée les résultats de l'analyse documentaire faisant l'objet d'un mémoire (Côté, 1998) rédigé 
dans le cadre d'un programme de maîtrise à l'École de service social de l'Université d'Ottawa. La méthodologie adoptée dans cette étude comporte une recension des écrits faite sous forme d'analyse documentaire, qui vise à vérifier et à établir où en est la recherche sur la compréhension qu'ont les intervenantes des diverses formes de deuils vécues par les femmes dans notre société. L'analyse documentaire implique les étapes suivantes: localiser les écrits pertinents, évaluer leur crédibilité et leur représentativité, explorer leur message et procéder à la recherche de toutes les pistes susceptibles de fournir des informations pertinentes (Cellard 1997 : 252).

Divers points de repère ont servi au choix des textes. D'abord ils devaient porter soit sur la femme, soit sur son vécu. Ces textes devaient également préciser la façon dont la perte et le travail de deuil étaient interprétés. Plusieurs des écrits choisis dépassent les balises de la définition du deuil proposée par la littérature classique. Les outils d'intervention et les stratégies de guérison suggérés dans les textes ont aussi influencé leur sélection. Les deux chapitres d'analyse du mémoire s'appuient donc sur deux études empiriques, trois textes académiques, trois textes centrés sur l'intervention, deux témoignages et deux poèmes (Côté 1998). Le présent article n'offre qu'un abrégé des résultats saillants qui se dégagent des écrits choisis.

\section{Thématique de recherche}

"Comment les femmes vivent-elles le travail de deuil? Comment comprendre et décrive le processus de guérison? Quel apport significatif peut-on tirer de l'analyse de perspectives non-conventionnelles?"
Nous nous pencherons d'abord sur certaines questions spécifiques par rapport au deuil. Comment les femmes vivent-elles le travail de deuil? Comment comprendre et décrire le processus de guérison? Quel apport significatif peut-on tirer de l'analyse de perspectives non-conventionnelles? Quelles implications pour l'intervention sociale se dégagent de cette analyse?

La définition du deuil incluant la dimension de la perte est difficile à cerner, étant donné que différents courants théoriques ont exploré la question avec des interprétations fort diverses. La 
"C'est précisément ce passage d'un état à l'autre qui nous intéresse."
"Il importe donc de porter un regard sur les résultats des études s'appuyant sur une variété d'approches, par exemple, les perspectives sociologiques, religieuses, spirituelles et anthropologiques." perte est définie comme « un vide, rempli de sentiments terrifiants : une haine brûlante, un désespoir grésillant, une colère qui déchire » (Roberts 1983 dans Kramarae et Treichler 1991). Une perte peut ainsi inclure la mort, une séparation ou des changements entraînant une souffrance. La perte s'accompagne d'un processus semblable à celui du deuil et, en ce sens, peut être considérée comme une rupture. Ces ruptures peuvent survenir suite à diverses expériences : des changements, un cheminement personnel ou professionnel, l'écroulement d'un rêve, la fin d'une illusion, la déception d'une potentialité non-exploitée. Il s'agit d'épisodes de la vie pouvant occasionner des réactions intenses de souffrance. Par la suite, il peut y avoir un sentiment de libération de cette peine, de cette souffrance et un cheminement vers une acceptation, une résolution ou une croissance. C'est précisément ce passage d'un état à l'autre qui nous intéresse. Le travail de deuil est « le processus cognitif impliquant la confrontation à la restructuration des pensées à propos de l'expérience de la perte et des changements de l'environnement dans lequel l'endeuillée doit maintenant vivre " (Stroebe 1992-1993 : 33). Il comporte une série d'étapes émotives qui suivent une perte importante et qui permettent un réajustement et une intégration; l'individu va se souvenir des événements passés, exprimer ses émotions, accepter sa situation, s'ajuster à de nouvelles conditions et s'embarquer dans de nouvelles relations (Baker 1993 : 156).

De nombreux facteurs peuvent influencer le travail ou le processus de deuil. Les valeurs et les croyances personnelles, familiales, religieuses, spirituelles et socioculturelles en sont quelques-uns. D'un point de vue macrosociologique, les institutions, les lois, les politiques sociales ainsi que les systèmes et les réseaux sociosanitaires peuvent aussi influencer le vécu de perte et le travail de deuil. Il importe donc de porter un regard sur les résultats des études s'appuyant sur une variété d'approches, par exemple, les perspectives sociologiques, religieuses, spirituelles et anthropologiques. Voilà pourquoi nous explorerons brièvement des perspectives qui interprètent le vécu de perte et le travail de deuil comme un processus de guérison, où les étapes ne sont peut-être pas définies de la même façon ni aussi clairement. De plus, c'est 
cette distinction qui inspire l'utilisation d'approches alternatives pour l'analyse des écrits choisis.

En s'éloignant des catégories ou des grilles d'analyse traditionnelles, telles que les approches médicales, psychologiques ou psychiatriques basées sur le DSM-IV, on ouvre les portes à de nouvelles perspectives et peut-être même à d'autres interprétations plus holistiques, plus éclairantes sur la façon dont les femmes vivent ces moments intenses.

\section{Les perspectives psychologiques}

"Les pertes du passé affectent notre façon de faire face aux défis futurs. Dans un tel contexte, il n'y pas réellement de début ni de fin : tout est processus. ”
La psychologie est un domaine d'étude qui explore des variables mesurables qui peuvent influencer l'individu. Selon McNeil (1994 : 289), « la phénoménologie du deuil implique une expérience globale qui concerne autant la psyché que le soma avec l'adoption d'attitudes comportementales".

L'interprétation de certains auteurs situe le deuil en fonction des réactions des personnes aux transitions apportées par la vie. Martin et Elder (1993 : 73) expliquent qu'avec chaque changement, il y a un choix à faire : le reconnaitre ou l'ignorer. Les pertes du passé affectent notre façon de faire face aux défis futurs. Dans un tel contexte, il n'y pas réellement de début ni de fin: tout est processus.

Wylie (1996: 124) offre également une perspective sur le potentiel de vivre des deuils face à des pertes autres que la mort d'un être cher. Elle explique que la perte de son "soi ", à travers des relations difficiles peut être très sérieuse pour la femme. Elle écrit aussi qu'une grande insécurité peut survenir lorsque les buts, les rêves ou les relations sont menacés par la perte d'un conjoint, le départ des enfants, etc. Les femmes investissent aussi leur "soi " dans des rôles multiples et quotidiens au sein de leur famille et dans le contexte de leur carrière ou de leur retraite; s'il survient un problème, une rupture ou une désillusion, il est extrêmement ardu de s'en remettre car une partie de leur "soi " s'éteint 
également (Wylie 1996: 136). De plus, les femmes transigent étroitement et plus fréquemment avec les pertes dites maternelles telles que l'infertilité, la grossesse, le décès d'un enfant ou les troubles de développement de l'enfant (Simonds 1988 : 149).

Selon l'interprétation psychologique, le deuil "atypique", " chronique " ou " pathologique " est caractérisé par des réactions tardives, soit parce qu'elles persistent à long terme ou parce qu'elles surgissent longtemps après la perte (Parkes 1996 : 110). Les personnes qui ont des réactions de deuil "pathologiques" démontrent parfois des symptômes physiologiques, par exemple des troubles cardiaques ou respiratoires (Parkes 1996 : 114).

\section{Les perspectives socio-anthropologiques}

«... chaque civilisation et temps de l'histoire ont trouvé face à la mort leurs stratégies, leurs modes d'apprivoisement, leurs rituels sacrés et profanes pour transcender ce point aveugle, pour permettre l'absolution collective de l'échéance mortelle et lui donner sens »."
L'anthropologie, pour sa part, tente de replacer l'individu dans son contexte socioculturel et d'identifier les caractéristiques de sa société et de sa culture d'appartenance. Par exemple, il s'agira dans ce cas d'observer les coutumes, les mœurs, les croyances et les systèmes sociaux de cultures habituellement non soumises aux influences occidentales. Les écrits anthropologiques sur le deuil décrivent les forces sociales qui ont un impact sur la manière dont le deuil est vécu chez les membres d'une société en particulier.

La façon dont la mort est perçue dans une société influence fortement le vécu lors du travail de deuil. Lasvergnas (1985: 692) l'explique ainsi : "chaque civilisation et temps de l'histoire ont trouvé face à la mort leurs stratégies, leurs modes d'apprivoisement, leurs rituels sacrés et profanes pour transcender ce point aveugle, pour permettre l'absolution collective de l'échéance mortelle et lui donner sens ».

Les auteurs Catlin (1992) et Rosenblatt (1976) démontrent que l'étude de différents groupes culturels permet de vérifier l'influence d'une multitude de facteurs qui ont le potentiel d'agir sur le vécu des personnes. Dans une étude anthropologique se fondant sur les récits de vie de personnes mourantes dans deux 
"...il y a des concepts tels le temps et l'espace qui, malgré leur grande importance, ne sont pas retenus dans des études de type empirique. "

"...les analyses

féministe et spirituelle ouvrent la voie à une autre analyse $d u$ processus de guérison des femmes. " régions différentes (rurales et urbaines) d'une même province, Des Aulniers (1997) explore leurs réactions face à la mort qui approche. Elle dégage des thèmes qui émanent du discours des participantes et des participants : l'émotion et ses tabous, le travail, l'espace, le changement, le temps, les facteurs historiques, les tensions et la perception du statut social de la maladie. En effet, elle démontre qu'il y a des concepts tels le temps et l'espace qui, malgré leur grande importance, ne sont pas retenus dans des études de type empirique.

Osterweis et al. (1984: 204) expliquent que des variables socioculturelles telles que la classe socioéconomique et des facteurs sociopolitiques peuvent avoir une influence sur la façon dont le groupe décide des normes qui régissent et guident les réactions "normales » au deuil. Pour certains groupes il est plus acceptable de réagir par des symptômes somatiques, alors que pour d'autres, il s'agit de s'abandonner entièrement à la religion et aux pratiques prescrites par celle-ci.

Il se dégage de ces perspectives psychologiques et socioanthropologiques que le vécu de deuil peut être analysé selon divers schèmes de référence. Certaines croyances et normes peuvent influencer la façon dont les réactions face aux pertes et au deuil peuvent être interprétées et vécues. Par ailleurs, les analyses féministe et spirituelle ouvrent la voie à une autre analyse du processus de guérison des femmes.

\section{Le processus de guérison $\square$ : analyse féministe}

Comme les valeurs, les normes, les lois et les politiques sociales ont un impact direct sur nos vies, nous cherchons à déterminer jusqu'à quel point les pertes, les deuils, le travail de deuil et le processus de guérison des femmes sont reliés à leurs expériences en tant que femmes dans une société donnée. Nous présentons dans cette section un résumé des résultats de l'analyse de textes liés au vécu des femmes et replacés dans le contexte de l'oppression qu'elles vivent au quotidien. Ceux-ci sont regroupés sous trois 
volets liés à la condition des femmes : la violence, la féminisation de la pauvreté et la santé.

\section{La violence faite aux femmes}

Une littérature importante (Bourgon et Corbeil 1990) démontre que malgré l'entrée relativement récente des femmes dans l'arène politique et économique, le " vrai " pouvoir est toujours entre les mains des hommes. Ce sont eux qui établissent les normes et les valeurs reconnues, qui définissent les critères de santé mentale et de violence et qui façonnent les lois et les politiques. Malgré plusieurs décennies de luttes féministes, ils ont encore souvent tendance, lorsqu'il s'agit de violence, à parler de provocation de la part de la femme plutôt que de domination et de victimisation de cette dernière. "La société patriarcale accorde aux hommes de nombreux avantages et privilèges qu'ils ne sont pas prêts à remettre en question" (Bilodeau $1990: 47$ ).

"Cette oppression engendre un isolement qui perpétue la négation des dimensions sociales, collectives et structurelles de la violence faite aux femmes...»
Cette oppression engendre un isolement qui perpétue la négation des dimensions sociales, collectives et structurelles de la violence faite aux femmes, comme le fait de refuser à ces dernières le soutien nécessaire pour se remettre de situations de violence et les outils essentiels qui pourront leur permettre de reprendre le contrôle et le pouvoir sur leur vie (Lacombe 1990 : 132).

Clark, Gordon et Enzle (1991) présentent une étude exploratoire des réactions de deuil «compliqué » de femmes ayant été violentées sexuellement pendant leur enfance. Les résultats font état d'un lien entre un passé ou un vécu de violence, les stratégies de survie de ces femmes et la façon dont elles vivent leurs deuils. S'appuyant sur une analyse féministe, ces auteurs démontrent que tout en se victimisant de l'abus et en intériorisant cette tragédie, la femme se responsabilise des actes d'abus. Comme la femme ne reprend pas le pouvoir dans cette situation, les réactions de deuil " compliqué " qu'elle aura par la suite perpétueront le cycle d'impuissance et l'empêcheront de faire l'apprentissage de nouvelles stratégies pour transiger avec d'autres pertes et deuils. Ces mêmes auteurs démontrent aussi que le deuil non résolu ou "compliqué " est encore aujourd'hui interprété par plusieurs comme une 
"Les thérapies de conscientisation, la validation, la prise de conscience du tabou et le partage des solutions, s'avèrent des voies à suivre pour l'intervention féministe. " mésadaptation chez la femme, alors que celle-ci est dépassée par le fait que ses pertes ne sont ni identifiées, ni reconnues, ni validées. Serait-ce parce que notre société responsabilise la victime pour l'abus qu'elle a vécu en privilégiant par exemple une approche "psychologisante "? Ne devrait-on pas plutôt décortiquer les pertes, vivre les histoires et les souffrances et explorer le côté positif de leurs stratégies?

Le texte de Fulmer (1987) porte un regard sur la possibilité d'entraide ou de kinship parmi les femmes. Les thérapies de conscientisation, la validation, la prise de conscience du tabou et le partage des solutions, s'avèrent des voies à suivre pour l'intervention féministe. N'y aurait-il pas avantage à promouvoir le partage entre les victimes d'abus? Doit-on collectiviser ces réactions afin de permettre un éveil pouvant mener à l'établissement de nouvelles stratégies de guérison et afin de cesser de faire de ce deuil légitime une "pathologie "?

\section{La féminisation de la pauvreté}

La femme célibataire à la tête d'une famille risque fort de se retrouver dans une situation d'extrême pauvreté (Robichaud et al. in Kérisit et St-Amand 1997 : 147). La vulnérabilité économique de la femme est un reflet de sa position désavantagée sur le marché du travail, ce dernier étant lui-même de plus en plus morcelé et discriminant. L'emploi précaire, l'iniquité salariale, le coût élevé des services de garde sont autant de facteurs qui s'ajoutent aux coupures budgétaires, à la cessation des paiements de transfert et à la destruction des structures sociales et qui touchent particulièrement les femmes (Kitchen 1997 : 111). La déstabilisation sociale et économique des femmes en est davantage accentuée.

Dans son étude sur les difficultés associées au travail de deuil au sein des familles monoparentales à faible revenu, Fulmer (1987) analyse les conditions de vie de certaines femmes dans notre société et leur impact sur la résolution de la perte et du travail de deuil. Les résultats démontrent que les proches, la famille étendue et la communauté procurent parfois un soutien très efficace. Par ailleurs, la fréquence et l'intensité des pressions environnementales négatives 
"L'auteur démontre que la jeune mère célibataire porte une accumulation de pertes, ce qui peut engendrer un sentiment de désespoir, amplifiant l'impact de la perte actuelle. » apportent à la famille à faible revenu plus d'occasions de vivre des deuils et en même temps, enlèvent en partie les capacités de vivre ce deuil. Puisque leurs pertes matérielles sont si critiques, les effets psychologiques des mesures de compensations ne sont pas reconnus, ce qui contribue à faire du deuil non-complété une norme chez cette population. Ce texte traite des enjeux et des conséquences de la pauvreté pour la jeune femme. L'auteur démontre que la jeune mère célibataire porte une accumulation de pertes, ce qui peut engendrer un sentiment de désespoir, amplifiant l'impact de la perte actuelle.

\section{La santé de la femme}

Plusieurs critiques féministes s'intéressent à la médicalisation comme processus de construction sociale des rapports entre les sexes. De Koninck (1995 : 33), par exemple, définit la gestion biomédicale comme suit : «l'adhésion à la définition biomédicale de la spécificité biologique des femmes et le contrôle biomédical des événements liés à cette spécificité; la reproduction en est le point de départ». Bacqué (1992) pour sa part explique que le système de santé exerce sur la femme un contrôle social accablant et opprimant; y joindre les "symptômes " de deuil ne fait que renforcer le pouvoir que détient sur elle la communauté médicale.

"Le danger réside dans le fait que la femme, déjà ciblée médicalement, devient une proie facile du système de "soins »." Le danger réside dans le fait que la femme, déjà ciblée médicalement, devient une proie facile du système de «soins ".

La santé mentale représente un second enjeu crucial pour la femme qui vit un deuil, surtout depuis que plusieurs intervenantes et intervenants (incluant les psychiatres et psychologues) associent davantage le deuil à la catégorie des désordres du choc posttraumatique du DSM-IV (McNeil 1994 :287). Clark, Gordon et Enzle (1991) démontrent que les participantes de leur étude faisant face à un diagnostic sérieux peuvent aussi vivre une perte. Ces auteurs soulignent que, même si elles sont en rémission ou guéries, ces femmes vivent néanmoins un deuil, qui peut être lié à la possibilité que la maladie provoque une remise en question de sa vie et de son vécu. Cette expérience peut s'avérer très difficile car elles doivent éventuellement faire face à d'autres expériences 
"...si l'on ne se donne pas le temps de vivre le cycle du deuil à un rythme naturel, certains symptômes surgiront un jour ou l'autre. En ce sens, Shore (1995a) suggère que les symptômes psychosomatiques sont des voix non entendues." traumatisantes. Cette crise peut être interprétée comme une réaction de deuil anormale ou «compliquée " alors qu'elles vivent un deuil de leur passé en plus de celui provoqué par leur maladie.

Shore (1995a) explique que l'histoire de la femme définit qui elle est et d'où elle vient. Elle introduit des concepts tels que le temps, le processus, l'écoute de soi, le repos, le "cure", pour finalement aboutir à l'équilibre retrouvé. Elle suggère que si l'on ne se donne pas le temps de vivre le cycle du deuil à un rythme naturel, certains symptômes surgiront un jour ou l'autre. En ce sens, Shore (1995a) suggère que les symptômes psychosomatiques sont des voix non entendues. Le concept de "Re-member", se souvenir et se reprendre en charge, explique les deux aspects du début du cheminement vers la guérison. Il faut d'abord se souvenir de ces parties que nous avons mises de côté pour de multiples raisons et ensuite écouter ces symptômes qui représentent des voix perdues et silencieuses appartenant aux aspects dissociés du moi.

Divers auteures et auteurs cités dans cette section sur la perspective féministe soulignent l'importance de reconnaître les vulnérabilités et les besoins spéciaux des femmes survivantes d'abus, sexuels ou autres, subis pendant leur enfance. Elles peuvent avoir vécu un traumatisme qui les rend sujettes à des deuils qualifiés de " compliqués » par la littérature classique. Transiger avec une telle situation en intervention exigerait que soient pris en considération le passé, les abus et les sentiments qui y sont associés. Quant aux femmes qui vivent des situations précaires, il s'agit de clarifier avec elles le rôle qu'elles assument dans leur système de proches ou de soutien et de discuter des relations de pouvoir à l'intérieur de ce même système.

Ensuite, il faut se rappeler la mise en garde relative aux pertes accumulées et aux deuils ignorés et non résolus, qui peuvent se traduire en symptômes physiologiques.Au-delà de ces symptômes, on peut aider la personne à reconnaitre la complexité de la situation et à se donner le temps de cheminer à son propre rythme. Il faut écouter les voix perdues et aider la personne à s'approprier les parties ignorées, non reconnues et non validées de son moi. Il s'agit d'écouter tous les aspects de l'histoire de la personne : ses émotions, son physique, son social, son sexuel, son cognitif, sa 
complexité, sans négliger son potentiel de guérison.

\section{Processus de guérison $\square$ : analyse spirituelle}

"...la guérison

spirituelle consiste à devenir libre de rêver, de manifester ses émotions, de visualiser, comme femme, un monde ayant une signification, avec la certitude que cette vision peut être réalisée..."
L'exploration du processus de guérison à partir d'une perspective spirituelle peut-elle apporter un complément d'information permettant de mieux apprécier la complexité, mais aussi la richesse possible de telles expériences? Cinq textes choisis dans le cadre de notre analyse documentaire insistent sur le rôle de la spiritualité dans le travail de deuil et le processus de guérison. De ces écrits, nous retenons les volets suivants : le mouvement de pendule entre la douleur et la réconciliation, l'importance de la culture et des croyances et enfin les stratégies de survie.

Selon les auteurs de ces textes, la guérison spirituelle consiste à devenir libre de rêver, de manifester ses émotions, de visualiser, comme femme, un monde ayant une signification, avec la certitude que cette vision peut être réalisée dans ce monde. La guérison représente alors le dépouillement d'années d'abus et d'oppression qui nous empêchent de nous voir l'une et l'autre dans toutes nos grandeurs, nos formes et nos couleurs posées comme sacrées (Sauvé 1997).

\section{Le mouvement de pendule entre la douleur et la réconciliation}

"...en vivant le deuil, on travaille à donner un nouveau sens et une nouvelle direction à sa vie. »
Le texte de Milman (1995), décrit son cheminement en tant qu'enfant de parents survivants de l'Holocauste. Elle postule qu'en composant avec le désarroi, nous nous engageons face à la douleur et nous apprenons à la dépasser.Vivre le deuil permet de travailler à soulager ou à amenuiser la détresse et l'angoisse de la souffrance, de se transformer afin de trouver de nouvelles façons de vivre et de rétablir une synchronicité avec soi-même. Cela corrobore les propos d'Attig (1996: 143) au sujet de la douleur : en vivant le deuil, on travaille à donner un nouveau sens et une nouvelle direction à sa vie. Ce passage du deuil à la guérison permet de redevenir entière, de faire à nouveau partie du monde. Attig (1996 : 135) 
soutient également que nous avons toutes une toile nous reliant au monde, tissée avec des fils qui représentent nos particularités émotives, physiologiques, pratiques, sociales, intellectuelles et spirituelles. Dans la guérison, il faut parfois réajuster les fils ou tisser de nouveaux liens d'attache aux autres et au monde qui nous entoure.

\section{L'importance de la culture et des croyances}

Bodnar (1994) partage son expérience en tant qu'intervenante auprès de personnes autochtones à tendance suicidaire ayant perdu des membres de leur famille suite à un suicide. Elle dresse d'abord un aperçu historique et politique du vécu des autochtones. Bourgault (1985:30) explique que toute intervention auprès de ces derniers doit débuter par une exploration de leur culture. Traditionnellement, les autochtones vivent en harmonie avec la nature, dans un climat communautaire profondément humain. Leur ambition est de se comprendre, de voir grandir les enfants et

"L'autochtone vit de liberté. Sa vision du monde est unifiée. Ce souci de l'unité et de l'harmonie provient de sa spiritualité; son pouvoir est presque infini..." de s'épanouir en tant que peuple. L'autochtone vit de liberté. Sa vision du monde est unifiée. Ce souci de l'unité et de l'harmonie provient de sa spiritualité; son pouvoir est presque infini car il reconnaît l'esprit dans toutes les situations de la vie quotidienne et les sens qui l'entourent.

Cela dit, il importe de proposer des liens entre ces traditions et ces croyances et ce que l'auteure du texte a partagé de leur souffrance. Le concept de la souffrance transgénérationnelle est considéré comme très important. Toutefois, la connexion entre les générations n'est pas évidente et la souffrance est vécue en silence. De cette façon, les mots sont cachés et les souvenirs oubliés. De plus, l'idée d'explorer les effets de l'oppression de génération en génération est enrichissante : elle peut expliquer les effets de l'oppression chez d'autres groupes cibles tels que les femmes et d'autres cultures minoritaires. La souffrance permet de comprendre l'impact social de nos croyances sur notre manière de transiger avec le présent.

Les résultats de l'étude de Nathanson (1995) ajoutent à ce fil conducteur de l'influence des croyances et de l'expérience de la 
"...les femmes pour qui la spiritualité est importante, expriment qu'elles $y$ ont puisé force et courage au cours $d u$ processus de guérison. C'est une façon de comprendre et d'apprécier l'impact d'une vision spirituelle sur le quotidien des femmes.» douleur. Cette dernière préconise que la femme qui vit un divorce vit une perte. Cependant, la personne qu'elle a aimée, et qu'elle aime peut-être encore, n'est pas décédée. La perte ici est celle d'un rêve. Le déséquilibre créé par cette perte est différent et ses effets peuvent aussi être interprétés différemment par la personne. De plus, les résultats démontrent que les femmes pour qui la spiritualité est importante, expriment qu'elles y ont puisé force et courage au cours du processus de guérison. C'est une façon de comprendre et d'apprécier l'impact d'une vision spirituelle sur le quotidien des femmes.

\section{Les stratégies $\square$ : se souveni,rle silence, le symbolisme, les rythmes et les techniques}

Plusieurs auteurs explorent les stratégies utilisées pour faire face aux problèmes liés au deuil et aux pertes. Milman (1995), présentée antérieurement, propose la stratégie de se souvenir, notamment en rassemblant des photos de sa famille étendue et en reconstituant son histoire familiale. Voilà une façon de se rappeler, de ne pas oublier qu'il y a un précédent à la guérison.

"Le silence occupe une place importante dans la spiritualité. »
Le silence occupe une place importante dans la spiritualité. Shore (1995b) avance que le silence et les «non-dits » permettent un rythme et une individualité au vécu des pertes. De plus cette auteure aborde les thèmes de l'influence que peut avoir la nature sur la force, l'espoir et le pouvoir de la personne. À ce sujet, Milman (1995) rajoute que le "non-faire " et la "non-action " dans le processus de guérison donnent le temps de rétablir l'équilibre et la force personnelle.

Cette dernière explore aussi le thème de l'art comme thérapie dans la perspective globale du processus de réintégration et de régénération de l'organisme humain. L'art permet à l'individu non seulement de participer au niveau d'un échange cognitif, mais de s'engager par les diverses fonctions sensorielles et perceptives dans une expérience par laquelle le vécu phénoménologique et affectif est mis en valeur (Grégoire 1988 : 946). Le message peint, écrit ou dansé échappe facilement aux interdits 
"Les concepts de recentrage, pour la personne qui recherche la guérison, de même que l'abandon pour celle qui guérit, nécessitent le passage à un autre état de conscience." grâce aux aspects ludiques et permissifs de l'art. À travers son art, l'auteure intègre ses pertes personnelles et familiales (Anderson et Gold 1998 : 17). Cette stratégie se rapproche du concept de prière comme l'explique Dossey (1994). Les concepts de recentrage, pour la personne qui recherche la guérison, de même que l'abandon pour celle qui guérit, nécessitent le passage à un autre état de conscience.

Sauvé (1997) rapporte les résultats d'une recherche faite auprès d'intervenantes en violence visant à identifier les diverses techniques découvertes par les femmes dans leur processus de guérison. Celles-ci ont partagé leurs histoires personnelles, leurs idées, les approches et les outils qui ont contribué à intégrer la spiritualité à la pratique avec des femmes victimes de violence. Voici le fruit de ces découvertes et les concepts qui s'en dégagent :

- le sentiment de sécurité : aider la personne à trouver une aire de repos et de paix intérieure pour se recentrer sur elle-même;

- le travail avec le désespoir et la dépression : il faut trouver la source sacrée pour guérir sa colère et sa haine;

- le travail avec la respiration, l'énergie et la méditation : en prenant conscience de la présence des sentiments et à partir de la respiration, on peut trouver l'endroit où l'énergie est bloquée;

- la prière et les guides spirituels : la prière est un partage qui permet d'appeler les forces de l'Univers pour soutenir une personne. Un guide spirituel peut être un ange spirituel à qui l'on parle, à qui l'on pose des questions ou auprès de qui l'on invoque des explications;

- les saisons et les cycles de la vie : un travail avec les symboles est possible. On peut recadrer les cycles des événements de façon créative. Des symboles peuvent être créés pour célébrer les transitions et les rites de passage;

- la guérison autochtone : les femmes autochtones vivent leurs guérisons dans leurs traditions et dans leurs cérémonies. C'est une reconnaissance des liens entre les aspects physique, émotif, mental et spirituel. Elles peuvent renouer avec le cercle de guérison, la roue médicinale, les " elders ", les " powwows " et le "vision quest »; 
"...il est essentiel de connaître la culture, les croyances et les valeurs d'une personne dans le contexte d'une relation d'aide. Par exemple, une personne autochtone peut vivre son deuil selon une philosophie multigénérationelle où la source de l'être est sa lumière. »
- le recadrage des images négatives et des images positives: l'intervenante encourage la femme à se dépouiller des images et des perceptions négatives, à cultiver ses propres racines, ses croyances et sa sagesse à l'intérieur de son histoire personnelle.

Les saisons et les cycles représentent des processus naturels d'équilibre. On peut ainsi explorer les interprétations de chacune et découvrir avec elle le sens de sa douleur et de son cheminement. Sauvé (1997) souligne qu'il est essentiel de connaitre la culture, les croyances et les valeurs d'une personne dans le contexte d'une relation d'aide. Oar exemple, une personne autochtone peut vivre son deuil selon une philosophie multigénérationelle où la source de l'être est sa lumière. Pour elle la guérison sera possible si l'on favorise le mouvement entre le fait de se souvenir et d'oublier. Elle doit pouvoir se retrouver et raconter son histoire. Cette liberté d'expression, de créativité, cette permission de vivre l'émotion et d'avoir une histoire sont aussi symboliques que le temps consacré à vivre ces expériences.

Les outils d'intervention présentés dans cette section démontrent l'importance de la connexion corps-esprit où la sécurité, la respiration, la méditation et les énergies peuvent avoir un impact positif sur le cycle naturel de guérison. Au cours de ce cheminement, la personne peut recadrer ses interprétations, ses images et ses symboles. L'intervenante peut créer un espace où la personne peut vivre sans pression ni attentes à travers ses expériences.

\section{Conclusion}

Le but de cette analyse documentaire était d'explorer, dans certains textes choisis, le tissage de liens entre le vécu de pertes, le travail de deuil et le processus de guérison chez les femmes. Les deux perspectives d'analyse retenues ont permis de regarder sous plusieurs angles, l'expérience des femmes par rapport à leurs pertes et à leurs deuils. Ce faisant, nous avons relevé certaines pistes 
"Il ressort des textes que les difficultés des femmes qui vivent des deuils s'expliquent davantage par l'oppression dont elles sont victimes que par un manque de capacités individuelles. » pour faciliter le processus de guérison. L'analyse féministe a replacé les expériences des femmes vivant des pertes et des deuils dans leur contexte familial, historique et sociopolitique. Plusieurs des idées préconçues face aux deuils pathologiques ont été remises en question à la lumière de l'analyse de la condition de la femme dans notre société. L'analyse spirituelle du processus de guérison est venue par ailleurs éclairer des thèmes tels que la philosophie multigénérationnelle et le sens de la souffrance, la douleur, les croyances, les symboles et les cycles que vivent les femmes.

Les principes d'analyse féministe ont permis de concrétiser les problèmes structurels du vécu des femmes; des liens ont été établis entre les trois volets importants de la condition de la femme, soit la violence, la pauvreté et la santé. Dans la littérature traditionnelle, les problèmes que vivent les femmes sont souvent isolés et analysés individuellement de sorte que tous les symptômes mènent à un diagnostic de dépression ou de deuil compliqué. Nous avons démontré que le deuil " compliqué » ou non résolu peut s'expliquer sans avoir recours à la pathologie. Il ressort des textes que les difficultés des femmes qui vivent des deuils s'expliquent davantage par l'oppression dont elles sont victimes que par un manque de capacités individuelles. L'analyse féministe a aussi renforcé la notion que les stratégies actuelles des femmes, par exemple l'appui des proches et le partage de vécus communs, constituent une façon de collectiviser leur souffrance.

Les principes d'analyse spirituelle ont quant à eux démontré que vivre les émotions associées à la perte et au deuil peut contribuer au développement d'une grande force. L'ouverture à la douleur et à la souffrance peut mener à la guérison.

Enfin, les perspectives féministe et spirituelle se rejoignent en ce sens que leur nature alternative permet l'exploration d'autres visions et ouvre la voie à de nouvelles interprétations de phénomènes anciens. L'analyse axée sur ces deux perspectives se veut plus globale et inclut la spiritualité comme composante essentielle à la guérison. Le recours à une spiritualité explorée mais peu définie est peut-être la force qui a permis aux femmes de survivre à des décennies d'oppression. Leurs stratégies et leurs transformations en tant que femmes font d'elles des êtres généreuses, 
"Les textes étudiés démontrent qu'en leur for intérieur, les femmes possèdent les forces nécessaires pour affronter les épreuves, et que la personne est elle-même la meilleure guide vers son rétablissement et vers sa croissance." conscientes de leur environnement et enrichies par la force et le pouvoir de la douleur transformée en guérison.

Les résultats de notre analyse peuvent se classer en quatre parties, tel qu'illustré dans le cercle de synthèse suivant (voir le cercle synthèse). La première partie dresse un bilan du vécu des femmes par rapport à leurs pertes et à leurs deuils; cela inclut la violence, la souffrance, la douleur, l'accumulation des pertes et la peur. La deuxième partie présente les conséquences de ce vécu : la rupture, la déstabilisation, la désillusion, le vide et le déchirement. Le processus de guérison porte une attention spéciale aux concepts suivants : le temps, l'espace, le rythme, le va-et-vient, les émotions, l'ouverture de soi, le lien corps-esprit, son histoire, le pouvoir et le système des proches. Les résultats de ce vécu et de ce processus sont l'évolution, la guérison, l'équilibre, l'acceptation, l'interaction, l'harmonie, la transformation et la croissance.

Les textes étudiés démontrent qu'en leur for intérieur, les femmes possèdent les forces nécessaires pour affronter les épreuves, et que la personne est elle-même la meilleure guide vers son rétablissement et vers sa croissance. Il s'agit d'être respectueuses de leurs rythmes et d'être conscientes de leur potentiel de guérison. Le rôle de l'intervenante est d'encourager la personne à se laisser aller et à suivre sa propre croissance. Cette croissance peut conduire à la formulation de leçons de vie, à la sagesse, à la créativité, à la reconnaissance des émotions, à la capacité d'apprivoiser l'inconnu et de s'y abandonner avec confiance.

La résolution se fait d'une façon cyclique où l'ordre, l'intensité et la durée des phases sont variables. Le passage du deuil à la guérison se produit lorsqu'on change sa façon d'appréhender la perte subie de telle sorte que les limites sont recadrées de façon positive et les problèmes deviennent des défis. La transformation de la perte implique que la personne replace la perte dans un contexte de croissance, de cycles de vie et apprivoise la notion de deuil en tant qu'expérience humaine qui unifie plutôt qu'aliène. 


\section{Figure 1-Cercle-Synthèse}

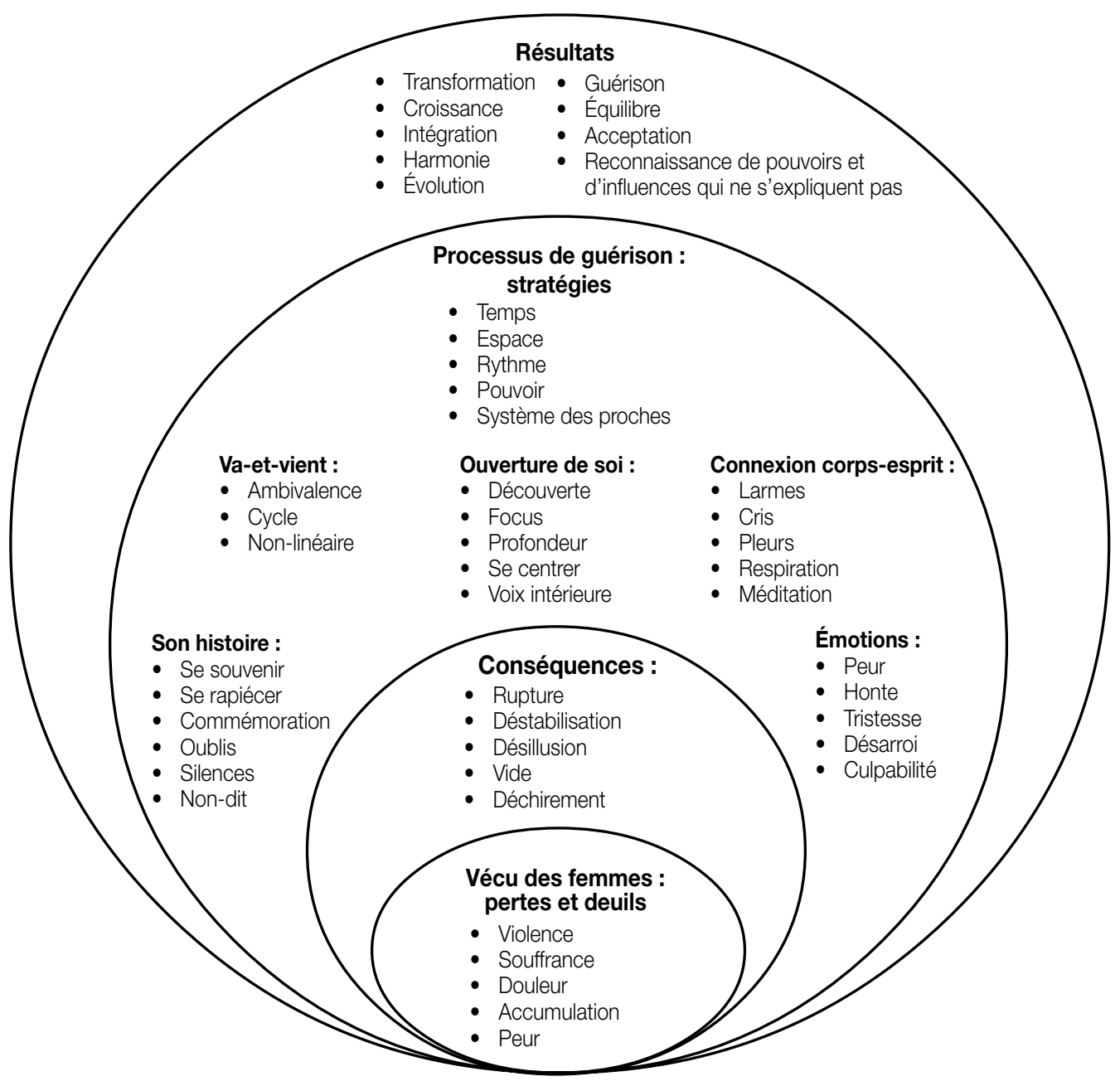

\section{Bibliographie}

ANDERSON, L. et K. GOLD (1998). «Creative Connections : The Healing Power of Women's Art and Craft Work », Women E Therapy, 21 (4), 15-36. 
ATTIG,Thomas (1996). "Relearning Ourselves : Grief and Personal Integrity ", in How we Grieve : Relearning the World, New York, Oxford University Press, 128-160.

BACQUÉ, Marie-Frédérique (1992). Le deuil à vivre, Paris, Éditions Odile Jacob.

BAKER, Robert L. (1993). The Social Work Dictionary, 3e éd., NASW Press.

BILODEAU, Dominique (1990). «L'approche féministe en maison d'hébergement : quand la pratique enrichit la théorie ", Pratiques féministes, 3 (2), 45-55.

BODNAR, Ana (1994). «Bereavement as Healing in First Nations Communities : The Importance of Remembering ", Luc BESSETTE (éd.), Le deuil comme processus de guérison, Montréal, MNH.

BOUR GAULT, Luc (1985). L'héritage sacré des peuples amérindiens, Ottawa, Les éditions de Mortage.

BOUR GON, Michèle et Christine COREIL (1990). «Dix ans d'intervention féministe au Québec : bilan et perspective ", Santé mentale au Québec, X.V. 1, 205-222.

CATLIN, George (1992). "The Role of Culture in Grief », The Journal of Social Psychology, 133(2), 173-184.

CELLARD, André (1997). "L'analyse documentaire ", Jean Poupart (dir.), La recherche qualitative : enjeux épistémologiques et méthodologiques, Montréal, Gaëtan Morin.

CLARK, G., C. GORDON and S. ENZLE (1991). "Complicated Grief Reactions in Women Who Were Sexually Abused in Childhood ", Journal of Psychological Oncology, 8(4), 87-97.

CÔTÉ, Geneviève (1998). «Le processus de guérison des femmes : leurs pertes, leurs deuils et leur croissance ", mémoire de maîtrise en service social, sous la direction de Nérée St-Amand, Ottawa, Université d'Ottawa (non publié).

De KONINCK, Maria (1995). "La gestion biomédicale du corps des femmes : un progrès? ", Bouchard L. et D. COHEN (dir.), Médicalisation et contrôle social, Montréal, ACFAS.

Des AULNIERS, Annick (1997). Itinérances de la maladie grave : le temps des nomades, Montréal, L'Harmattan Inc.

DOSSEY, Larry (1994). "What is Healing? ", in Healing Words : The Power of Prayer and The Practice of Medicine, New York, Harper San Francisco.

FULMER, Richard H. (1987). "Special Problems of Mourning in Low-Income Single-Parent Families ", Family Therapy Collections, 23, 19-37.

GRÉGOIRE, Pierre (1988). "Art et thérapie ", J. DUFRESNE, F. DUMONT et Y. MARTIN (dir.), Traité d'anthropologie médicale: L'Institution de la santé et de la maladie, Québec, Presses de l'Université du Québec, 941-956.

GUYON, Louise et Louise NADON (1990). «Le mouvement féministe et la santé mentale : que reste-t-il de nos amours? ", Santé mentale au Québec, XV 1, 7-28.

KÉRISIT, Michèle et Nérée ST-AMAND (1997), « Community Strategies for Surviving and Resisting the Cuts ", RALPH, D., A. RÉGIMBALD et N. ST-AMAND (dir.), Open for Business, Closed to People, Halifax, Fernwood Publishing.

KITCHEN, Brigitte (1997). «'Common Sense'Assaults on Families », RALPH, D.,A. RÉGIMBALD et N. ST-AMAND (dir.), Open for Business, Closed to People, Halifax, Fernwood Publishing.

KRAMARAE, et TREICHLER (1991). A Feminist Dictionary, Boston, The University of Illinois Press.

LACOMBE, Madelaine (1990). Au grand jour, Montréal, Éditions Remue-méninge. 
LASVER GNAS, Isabelle (1988). "La vie et la mort », J. DUFRESNE, F.DUMONT etY. MARTIN (éds), Traité d'anthropologie médicale: L'Institution de la santé et de la maladie, Québec, Presses de l'Université du Québec, 691-708.

MARTIN, Karen et Sandra ELDER (1993). "Pathways Through Grief : A Model of the Process ", in Personal Care in an Impersonal World: A Multidimensional Look at Bereavement, New York, Baywood Publishing Company Inc.

McNEIL, John S. (1994). «Bereavement and Loss », in Encyclopedia of Social Work, $19^{e}$ éd., New York, National Association of Social Workers, 284-290.

MILMAN, Isa (1995). "Within and Despite The Fiery Crucible of Memory; Healing and the Holocaust », Luc BESSETTE (éd.), Le deuil comme processus de guérison, Montréal, MNH.

NATHANSON, Irene G. (1995). « Divorce and Women's Spirituality ", Journal of Divorce and Remarriage, 22 (3-4), 179-188.

OSTERWEIS, M., F. SOLOMON et M. GREEN (1984). Bereavement : Reactions, Consequences and Care, Washington, National Academy Press.

PARKES, Collin Murray (1996). Bereavement: Studies of Grief in Adult Life, 3e éd., New York, Routledge.

ROSENBLATT, Paul C. et al. (1996). Grief and Mourning : in a Cross Cultural Perspective, U.S.A., HRAF Press.

SAUVE, Juanita (1997). «Healing the Spirit from the Effects of Abuse : Spirituality and Feminist Practice with Women who have been Abused ", thèse de maitrise en service social, Ottawa, Carleton University.

SHORE, Lesley Irene (1995a). "Healing ", in Tending Inner Gardens : The Healing Art of Feminist Psychotherapy, New York, The Haworth Press, 31-45.

SHORE, Lesley Irene (1995b). "Re-Membering ", in Tending Inner Gardens : The Healing Art of Feminist Psychotherapy, New York, The Haworth Press, 60-72.

SIMONDS, Wendy (1988). "Confessions of Loss : Maternal Grief in 'True Story', 1920-1985 », Gender and Society, 2(2), 149-171.

STEVENSON, Robert G. et al. (1998). "Document on Violence and Grief », Omega, 36(3), 259272.

STROEBE, Margaret (1992). "Coping with Bereavement : A Review of the GriefWork Hypothesis », Omega, 26(1), 19-42.

WYLIE, Betty Jane (1996). Life's Losses : Living through Grief, Bereavement and Sudden Change, Toronto, MacMillan Canada.

\section{Note}

1. L'auteure tient à exprimer sa profonde gratitude à son guide et directeur de mémoire, Nérée StAmand, pour son encouragement et son dévouement au cours de ce projet. Ses remerciements vont aussi à son amie et correctrice Pascale Guhur. 